\title{
Clinical features and drug sensitivity pattern of Klebsiella pneumoniae sepsis: A descriptive study in a level 2 neonatal care unit in India
}

\author{
Manjari Basu ${ }^{1}$ \\ Sri Lanka Journal of Child Health, 2017; 46(3): 259-261
}

\begin{abstract}
Objectives: To study the signs and symptoms of neonatal sepsis caused by Klebsiella pneumoniae and the antibiotic sensitivity pattern of Klebsiella pneumoniae in a level 2 neonatal unit in India.
\end{abstract}

Method: This descriptive study was conducted with neonates who were admitted in Sick Newborn Care Unit (level 2 neonatal care unit) and whose blood culture showed growth of Klebsiella pneumoniae in a study period of 1 year. The data was entered into a register and presented by descriptive statistics.

Results: Forty eight neonates were included in the study. Predominant presenting symptoms were abdominal distension (75\%), petechiae, purpura $(62.5 \%)$ and sclerema (50\%). Klebsiella pneumoniae was highly sensitive to tigecycline and meropenem.

Conclusions: Predominant presenting symptoms of neonatal sepsis caused by Klebsiella pneumoniae were abdominal distension, petechiae, purpura and early sclerema. Klebsiella pneumoniae was highly sensitive to tigecycline and meropenem.

DOI: http://dx.doi.org/10.4038/sljch.v46i3.8328

(Key words: Klebsiella pneumoniae, drug sensitivity)

\section{Introduction}

Blood stream infection by multi-drug resistant gram-negative bacilli is one of the most worrisome challenges in the field of health care especially in intensive care units. Their impact is mostly felt in neonatal care units. Here, outbreaks can result in high morbidity and mortality ${ }^{1}$. In recent years, Klebsiella pneumoniae is reported to ${ }^{1}$ College of Medicine and JNM Hospital, India
${ }^{*}$ Correspondence: basu.manjari@gmail.com
(Received on 31 December 2016: Accepted after
revision on 17 February 2017)
The authors declare that there are no conflicts of
interest
Personal funding was used for the project.
Open Access Article published under the Creative Commons Attribution CC-BY LC be the most common cause of nosocomial infection among all Gram-negative bacteria ${ }^{2}$.

\section{Objective}

To study the signs and symptoms of neonatal sepsis caused by Klebsiella pneumoniae and the antibiotic sensitivity pattern of Klebsiella pneumoniae in a level 2 neonatal unit in India.

\section{Method}

Study design: Descriptive study checked by STROBE checklist for observational study.

Setting: Study was conducted at Sick Newborn Care Unit (level 2 neonatal care unit) of College of Medicine and JNM Hospital, Kalyani, India. The unit has average bed occupancy rate 30 .

Study Period: One year ( $1^{\text {st }}$ March 2015 to $29^{\text {th }}$ February 2016).

Participants: Subjects of the study were neonates (age under 28 days) who were admitted in sick newborn care unit and whose blood cultures showed growth of Klebsiella pneumoniae.

Exclusion criteria:

1. Babies whose blood culture report showed growth of some other organism with Klebsiella.

2. Babies who were admitted with severe sepsis because initial clinical features were missed.

Data collection: The data was collected from case records.

Data analysis: The data was entered into a register and presented by descriptive statistics. All numeric values were expressed in exact number and percentages. Categorical variables were compared using $\mathrm{X}^{2}$ test. $\mathrm{P}<0.05$ was considered as statistically significant.

\section{Results}

Forty eight newborns were included in the study who were admitted in Sick Newborn Care Unit due to some cause other than sepsis during the study period. Their blood culture report showed only growth of Klebsiella pneumoniae. Of these 48 newborns, $24(50 \%)$ were preterm very low birth weight babies, $06(12.5 \%)$ were preterm low birth weight and $18 \quad(37.5 \%)$ were term appropriate for gestational age babies. No baby was term small for gestational age or preterm extremely low birth weight. Thirty $(62.5 \%)$ newborns were male and $18(37.5 \%)$ were female. Of these 48 newborns, $16(33.3 \%)$ were admitted 
due to perinatal asphyxia, $08(16.7 \%)$ for meconium aspiration syndrome, and $24(50 \%)$ were preterm very low birth weight babies. All of them were admitted on day 1 of life. Time of onset of symptoms is shown in Table 1 .

Table-1

Time of onset of symptoms $(n=48)$

\begin{tabular}{|l|c|}
\hline $\begin{array}{c}\text { Age when symptoms } \\
\text { occurred }\end{array}$ & Number (\%) \\
\hline Day 1 and Day 2 & $0(0)$ \\
\hline Day 3 & $12(25)$ \\
\hline Day 4 & $24(50)$ \\
\hline Day 5 to Day 7 & $08(16.7)$ \\
\hline After Day 7 & $04(08.3)$ \\
\hline
\end{tabular}

The symptoms and signs of neonates with blood culture positive for Klebsiella pneumoniae are shown in Table 2.

Table 2

Symptoms/Signs of neonates with blood culture positive for Klebsiella pneumoniae $(n=48)$

\begin{tabular}{|l|c|}
\hline \multicolumn{1}{|c|}{ Symptom/Sign } & Number (\%) \\
\hline Poor feeding & $20(41.7)$ \\
\hline Abdominal distension & $36(75.0)$ \\
\hline Petechiae, purpura & $30(62.5)$ \\
\hline Sclerema & $24(50.0)$ \\
\hline Gastrointestinal bleeding & $18(37.5)$ \\
\hline Shock & $20(41.7)$ \\
\hline Respiratory distress & $12(25.0)$ \\
\hline Vomiting & $08(16.7)$ \\
\hline
\end{tabular}

The drug sensitivity pattern of neonates with blood culture positive for Klebsiella pneumoniae are shown in Table 3.

Table 3

Drug sensitivity pattern of Klebsiella pneumoniae

\begin{tabular}{|l|c|}
\hline \multicolumn{1}{|c|}{ Drug } & $\begin{array}{c}\text { Neonates with Klebsiella } \\
\text { pneumoniae sensitive to } \\
\text { drug - Number (\%) }\end{array}$ \\
\hline Tigecycline & $48(100)$ \\
\hline Meropenem & $44(91.7)$ \\
\hline Amikacin & $40(83.3)$ \\
\hline Gentamicin & $40(83.3)$ \\
\hline Levofloxacin & $36(75.0)$ \\
\hline Imipenem & $08(16.7)$ \\
\hline Teicoplanin & $08(16.7)$ \\
\hline Cefotaxime & $0(0)$ \\
\hline Ceftriaxone & $0(0)$ \\
\hline Cefepime & $0(0)$ \\
\hline $\begin{array}{l}\text { Amoxicillin- } \\
\text { clavulanate }\end{array}$ & $0(0)$ \\
\hline $\begin{array}{l}\text { Piperacillin- } \\
\text { Tazobactum }\end{array}$ & $0(0)$ \\
\hline Linezolid & $0(0)$ \\
\hline Vancomicin & $0(0)$ \\
\hline
\end{tabular}

In this study Klebsiella pneumoniae was resistant to cefotaxime, ceftriaxone, cefepime, amoxicillinclavulanate, piperacillin-tazobactum, linezolid and vancomycin, had intermediate sensitivity to amikacin, gentamicin, levofloxacin, imipenem and teicoplanin and was highly sensitive to meropenem and tigecycline (Table 3 ).

Regarding the outcome, 12 (25\%) newborns expired and $36 \quad(75 \%)$ recovered. Clinical improvement was assessed by commencement of feeding. On an average, feeds were started on day 5 of treatment. However, intravenous antibiotics to which the bacteria were sensitive, were continued for 14 days.

\section{Discussion}

In a previous study neonatal sepsis caused by Klebsiella pneumoniae has been shown to present with non-specific features like fever or hypothermia, tachypnoea, apnoea and feed intolerance ${ }^{3}$. However, in our study, abdominal distension (75\%) and petechiae, purpura (62.5\%) were the most common presentations. Sclerema also developed in a high number of cases $(50 \%)$. In contrast, respiratory distress occurred at presentation in only $25 \%$ of babies. Most children developed symptoms on day $3(25 \%)$ or day 4 $(50 \%)$. The mortality $(25 \%)$ is almost the same or slightly higher than that found in other studies ${ }^{3,4}$. In other studies, Klebsiella pneumoniae was sensitive to piperacillin-tazobactum, cefotaxime and imipenem. However, in our setup, Klebsiella was resistant to all of them but highly sensitive to meropenem $(91.7 \%)$, tigecycline $(100 \%)$ and levofloxacin $(75 \%)$.

Currently available studies on multidrug resistant Klebsiella are predominantly based on intensive care units. There is a paucity of information about outbreaks in intermediate risk neonatal units ${ }^{5}$. Here we describe the clinical features and drug sensitivity pattern of Klebsiella pneumoniae infection in an intermediate risk, level 2 neonatal care unit (Sick Newborn Care Unit) ${ }^{6}$.

\section{Conclusions}

Predominant presenting symptoms of neonatal sepsis caused by Klebsiella pneumoniae were abdominal distension, petechiae, purpura and early sclerema. Klebsiella pneumoniae was highly sensitive to tigecycline and meropenem.

\section{References}

1. Giceffre M, Geraci DM, Bonura C, Saporito L, Graziano G, Insinga V et al. The increasing challenge of multidrug resistant gram-negative bacilli: Results of a 5 year active surveillance 
programme in a neonatal intensive care unit. Medicine (Baltimore). 2016; 95(10): e 3016 .

2. Ozsari T, Bora G, Ozdemir OMA, Kilic I. A neonatal septic arthritis case caused by Klebsiella pneumoniae: A case report. Journal of Clinical and Diagnostic Research 2016; 10(2):SDO1-SDO2. https://doi.org/10.7860/jcdr/2016/17651. 7230

3. Mai JY, Zhu ML, Chen C, HeXL, Lin ZL. Clinical characteristics of neonatal Klebsiella pneumoniae sepsis and its antibiotic sensitivity pattern of strains. Zhonqquo Dong Dai Er Ke Za Zhin. 2016; 12(9):700-3.

4. Zhou J, Li G, Ma X, Yang Q, Yi J. Outbreak of colonization by carbapenemase-producing Klebsiella pneumoniae in a neonatal intensive care unit: Investigations, control measures and assessment. American Journal of Infection Control 2015; 43(10):1122-4. https://doi.org/10.1016/j.ajic.2015.05.03 8

PMid: 26149749
5. Cassettari VC, Silveira IR, Balsama AC, Franco F. Outbreak of extended Spectrum beta-lactamase producing Klebsiella pneumonia in an intermediate risk neonatal unit linked to onychomycosis in a health care worker. Journal of Pediatrics 2006; 82(4):31626.

https://doi.org/10.2223/JPED.1519

PMid: 16912835

6. Committee on fetus and newborn. Level of neonatal care. American Academy of Pediatrics 2012; 130(3):587-97.

PMid: 22926177 